\title{
On Some Properties of the Heisenberg Laplacian
}

\author{
M. E. Egwe \\ Department of Mathematics, Universty of Ibadan, Ibadan, Nigeria \\ Email: murphy.egwe@mail.ui.edu.ng,me_egwe@yahoo.co.uk
}

Received May 11, 2012; revised June 18, 2012; accepted June 24, 2012

\section{ABSTRACT}

Let $I H_{n}$ be the $(2 n+1)$-dimensional Heisenberg group and let $\mathcal{L}_{\alpha}$ and $T$ be the sublaplacian and central element of the Lie algebra of $H_{n}$ respectively. For $\alpha=0$, denote by $\mathcal{L}_{0}:=\mathcal{L}$, the Heisenberg Laplacian and let $K \subset \operatorname{Aut}\left(H_{n}\right)$ be a compact subgroup of Automorphism of $H_{n}$. In this paper, we give some properties of the Heisenberg Laplacian and prove that $\mathcal{L}$ and $T$ generate the $K$-invariant universal enveloping algebra, $\mathcal{U}\left(\mathfrak{h}_{n}\right)^{K}$ of $H_{n}$.

Keywords: Heisenberg Group; Heisenberg Laplacian; Factorization; Universal Enveloping Algebra; Solvability

\section{Preliminaries}

The Heisenberg group (of order $n$ ), $H_{n}$ is a noncommutative nilpotent Lie group whose underlying manifold is $\mathbb{C}^{n} \times R$ with coordinates

$(z, t)=\left(z_{1}, z_{2}, \cdots, z_{n}, t\right)$ and group law given by

$$
\begin{aligned}
& (z, t)\left(z^{\prime}, t^{\prime}\right)=\left(z+z^{\prime}, t+t^{\prime}+2 \operatorname{Im}\left(z \cdot z^{\prime}\right)\right), \\
& \text { where } z \cdot z^{\prime}=\sum_{j=1}^{n} z_{j} \bar{z}_{j} \quad z \in \mathbb{C}^{n}, t \in I R .
\end{aligned}
$$

Setting $z_{j}=x_{j}+y_{j}$, then

$\left(x_{1}, x_{2}, \cdots, x_{n}, y_{1}, y_{2}, \cdots, y_{n}, t\right)$ forms a real coordinate system for $H_{n}$. In this coordinate system, we define the following vector fields:

$$
X_{j}=\frac{\partial}{\partial x_{j}}+2 y_{j} \frac{\partial}{\partial t}, Y_{j}=\frac{\partial}{\partial y_{j}}-2 x_{j} \frac{\partial}{\partial t}, T=\frac{\partial}{\partial t} .
$$

It is clear from [1] that $\left\{X_{1}, X_{2}, \cdots, X_{n}, Y_{1}, Y_{2}, \cdots, Y_{n}, T\right\}$ is a basis for the left invariant vector fields on $H_{n}$. These vector fields span the Lie algebra $\mathfrak{h}_{n}$ of $H_{n}$ and the following commutation relations hold:

$$
\left[Y_{j}, X_{k}\right]=4 \delta_{j k} T,\left[Y_{j}, Y_{k}\right]=\left[X_{j}, T\right]=\left[Y_{j}, T\right]=0 .
$$

Similarly, we obtain the complex vector fields by setting

$$
\left.\begin{array}{l}
Z_{j}=\frac{1}{2}\left(X_{j}-i Y_{j}\right)=\frac{\partial}{\partial z_{j}}+i \bar{Z} \frac{\partial}{\partial t} \\
\bar{Z}_{j}=\frac{1}{2}\left(X_{j}+i Y_{j}\right)=\frac{\partial}{\partial \bar{z}_{j}}-i z \frac{\partial}{\partial t}
\end{array}\right\} .
$$

In the complex coordinate, we also have the commutation relations

$$
\begin{aligned}
& {\left[Z_{j}, \bar{Z}_{k}\right]=-2 \delta_{j k} T,} \\
& {\left[Z_{j}, Z_{k}\right]=\left[\bar{Z}_{j}, \bar{Z}_{k}\right]=\left[Z_{j}, T\right]=[\bar{Z}, T]=0 .}
\end{aligned}
$$

The Haar measure on $I H_{n}$ is the Lebesgue measure $d z d \bar{z} d t$ on $\mathbb{C}^{n} \times R$ [2]. In particular, for $n=1$, we obtain the 3-dimensional Heisenberg group $H_{1} \cong R^{3}$ (since $\mathbb{C}^{n} \cong R^{2 n}$ ). Hence $H_{n}$ may also be referred to as $(2 n+1)$-dimensional Heisenberg group.

One significant structure that accompanies the Heisenberg group is the family of dilations

$$
\delta_{ \pm \lambda}(z, t)=\left( \pm \lambda z, \pm \lambda^{2} t\right), \lambda>0
$$

This family is an automorphism of $H_{n}$. Now, if $\sigma: \mathbb{C} \rightarrow \mathbb{C}$ is an automorphism, there exists an induced automorphism, $\tilde{\sigma} \in \operatorname{Aut}\left(H_{n}\right)$, such that

$$
\tilde{\sigma}(z, t)=(\sigma z, t) \text {. }
$$

For simplicity, assume that $\tilde{\sigma}$ and $\sigma$ coincide. Thus we may simply assume that if $\sigma \in \operatorname{Aut}\left(H_{n}\right)$, we have $\sigma(z, t)=(\sigma z, t)$.

\section{Heisenberg Laplacian}

An operator that occurs as an analogue (for the Heisenberg group) of the Laplacian

$\Delta=\sum_{j=1}^{n} \frac{\partial^{2}}{\partial x_{j}^{2}}$ on $R^{n}$ is denoted by $\mathcal{L}_{\alpha}$ where $\alpha$ is a parameter and defined by 


$$
\mathcal{L}_{\alpha}=-\frac{1}{2} \sum_{j=1}^{n}\left(Z_{j} \bar{Z}_{j}+\bar{Z}_{j} Z_{j}\right)+i \alpha T,
$$

where $\bar{Z}_{j}$ and $Z_{j}$ are as defined in (1) so that $\mathcal{L}_{\alpha}$ can be written as

$$
\mathcal{L}_{\alpha}=\frac{1}{4} \sum_{j=1}^{n}\left(X_{j}^{2}+Y_{j}^{2}\right)+i \alpha T .
$$

$\mathcal{L}_{\alpha}$ is called the sublaplacian. $\mathcal{L}_{\alpha}$ satisfies symmetry properties analogous to those of $\Delta$ on $R^{n}$. Indeed, we have that $\mathcal{L}_{\alpha}$

1) is left-invariant on $H_{n}$;

2) has degree 2 with respect to the dilation automorphism of $H_{n}$ and

3 ) is invariant under unitary rotations.

Several methods for the determination of solutions, fundamental solutions of (2) and conditions for local solvability are well known [3-5].

The Heisenberg-Laplacian is a subelliptic differential operator defined for $\alpha=0$ as $\Delta_{H_{n}}$ on $H_{n}$ and denoted by $\mathcal{L}$. It is obtained from the usual vector fields as

$$
\begin{aligned}
\mathcal{L}: & =\Delta_{H_{n}}:=\sum_{j=1}^{n} X_{j} \circ X_{j}+Y_{j} \circ Y_{j} \\
= & \sum_{j=1}^{n} \frac{\partial^{2}}{\partial x_{j}^{2}}+\frac{\partial^{2}}{\partial y_{j}^{2}}+4 y_{j} \frac{\partial^{2}}{\partial x_{j} \partial t}-4 x_{j} \frac{\partial^{2}}{\partial y_{j} \partial t} \\
& +4\left(x_{j}^{2}+y_{j}^{2}\right) \frac{\partial^{2}}{\partial t^{2}} .
\end{aligned}
$$

By a technique in [6], the operator $\mathcal{L}$ is factorized into two quasi-linear first order operators on $H_{n}$ as:

$$
A=\left(\frac{\partial}{\partial x_{j}}-i \frac{\partial}{\partial y_{j}}+\left(2 y_{j}-2 i x_{j}\right) \frac{\partial}{\partial t}\right)
$$

and

$$
A^{\dagger}=\left(\frac{\partial}{\partial x_{j}}+i \frac{\partial}{\partial y_{j}}+\left(2 y_{j}+2 i x_{j}\right) \frac{\partial}{\partial t}\right)
$$

so that

$$
\begin{array}{r}
\mathcal{L}=\left(\frac{\partial}{\partial x_{j}}-i \frac{\partial}{\partial y_{j}}+\left(2 y_{j}-2 i x_{j}\right) \frac{\partial}{\partial t}\right) \\
\left(\frac{\partial}{\partial x_{j}}+i \frac{\partial}{\partial y_{j}}+\left(2 y_{j}+2 i x_{j}\right) \frac{\partial}{\partial t}\right) .
\end{array}
$$

Introducing the Lie algebra structure, we have

$$
\left[A, A^{\dagger}\right]=-4 x \delta_{i j} T, \quad \text { where } T=\frac{\partial}{\partial t},
$$

indicating that the Heisenberg algebra is noncommutative and $\mathcal{L}$ is hypoelliptic [4]. We thus obtain an operator (which is a homogeneous element of $\mathcal{U}\left(\mathfrak{h}_{n}\right)$, the universal enveloping algebra of the Heisenberg group when $\mathfrak{h}_{n}$ is the Heisenberg algebra) [5] consistent with that of Hans Lewy [7]. In [2], it has been shown that none of the factors of $\mathcal{L}, A$ or $A^{\dagger}$ is solvable and as such, $\mathcal{L}$ is not solvable.

In this paper, we shall prove that $\mathcal{L}$ only possesses a trivial group-invariant solution and for $K \subset \operatorname{Aut}\left(H_{n}\right)$ a compact subgroup of $\operatorname{Aut}\left(H_{n}\right)$, we have that $\mathcal{U}\left(\mathfrak{h}_{n}\right)^{K}$ the $K$-invariant universal enveloping algebra of the Heisenberg group is generated by $T$ and $\mathcal{L}$.

Now, by a solution of a factor $A^{\dagger}$ say, we shall mean that if $x, y, t$ are independent real variables, and $\psi \in C^{1}$, such that $A^{\dagger}$ has a solution $u(x, y, t)$ in the neighbourhood $N_{t_{0}}$ of the point $\left(0,0, t_{0}\right)$, with $A^{\dagger}=\psi(x, y, t)$ then $\psi$ is analytic at $t=t_{0}$.

Definition 2.0. Let $\Omega$ be any open subset of $I R^{n}$, and $\alpha$ a number such that $0 \leq \alpha \leq 1$. A function $u$ on $\Omega$ satisfying

$$
\sup _{x, y \in \Omega, x \neq y} \frac{|u(x)-u(y)|}{|x-y|^{\alpha}}<+\infty
$$

is said to be uniformly Holder continuous with Holder exponent $\alpha$ if $0 \leq \alpha \leq 1$; when $\alpha=1$, they are called uniformly Lipschitz continuous. When $\alpha=0$, they are simply continuous and bounded. A function is said to be in $H^{1}$-space if its first partial derivatives satisfy a Holder condition with positive exponent, provided the distance of the points involved does not exceed 1.

Theorem 2.1. Let $\psi$ be a periodic real $C^{\infty}$-function which is analytic in no t-interval. Then there exists a $C^{\infty}$-function $F(x, y, t)$ determined by the derivative $\psi^{\prime}$ of $\psi$ such that

$$
\left(\frac{\partial}{\partial x}+i \frac{\partial}{\partial y}+2 i(x+i y) \frac{\partial}{\partial t}\right) \psi=F(x, y, t)
$$

has no $H^{1}$-solution, (no matter what open $(x, y, t)$-set taken as domain of existence).

For Proof, see [8].

Theorem 2.2. The Heisenberg Laplacian, $\mathcal{L}$ defined in (3) has no non-trivial group invariant solution.

Proof. Let $\varphi$ be a group-invariant solution of (3). We wish to show that $\varphi \equiv 0$. To do this, let

$(x, y, t) \mapsto \delta_{r}(x, y, t)$ be a map generated by the group of automorphisms, dilations $\left\{\delta_{r}: r>0\right\}$ where $r$ determines the growth or decay rate. If $\varphi: H_{n} \rightarrow R^{2 n+1}$ is defined by

$$
\varphi_{\delta_{r}}(x, y, t)=\left(r x, r y, r^{2} t\right),
$$

then obtaining the first and second order derivatives of 
$\varphi$ with respect to the independent variables we have

$$
\begin{aligned}
& \frac{\partial \varphi}{\partial x}=r, \frac{\partial \varphi}{\partial y}=r \quad \frac{\partial \varphi}{\partial t}=r^{2}, \frac{\partial^{2} \varphi}{\partial x \partial t}=0 \\
& \frac{\partial^{2} \varphi}{\partial y \partial t}=0, \frac{\partial^{2} \varphi}{\partial x^{2}}=0 \quad \frac{\partial^{2} \varphi}{\partial y^{2}}=0, \frac{\partial^{2} \varphi}{\partial t^{2}}=0 .
\end{aligned}
$$

Substituting these into (3), we obtain a trivial equation. But by Group-invariant method, we should obtain a system of ordinary differential equations of lower order (see [9] p. 185). Thus, there exists no non-trivial groupinvariant solution for $\mathcal{L}$. $\square$

Theorem 2.3. Let $K \subset U(n)$ be a compact subgroup of $U(n)$, then $\mathcal{U}\left(\mathfrak{h}_{n}\right)^{K}$ the $K$-invariant universal enveloping algebra of the Heisenberg group is generated by $T$ and $\mathcal{L}$.

Proof. Let $\mathcal{U}\left(\mathfrak{h}_{n}\right)^{K}$ be the algebra of $K$-invariant differential operators on $H_{n}$ and let $S\left(\mathfrak{h}_{n}\right)$ be the symmetric algebra generated by the set

$$
\left\{X_{1}, X_{2}, \cdots, X_{n}, Y_{1}, Y_{2}, \cdots, Y_{n}, T\right\} .
$$

We note that the derived action of $K$ on $\mathfrak{h}_{n}$ is given by

$$
\exp (k \cdot X)=k \cdot \exp (X), \quad X \in \mathfrak{h}_{n}, k \in K
$$

and $K$ acts on $\mathfrak{h}_{n}^{*}=\operatorname{Hom}_{I R}\left(\mathfrak{h}_{n}, R\right)$ via

$$
(k \cdot \alpha)(X)=\alpha\left(k^{-1} \cdot X\right), \alpha \in \mathfrak{h}_{n}^{*}
$$

and on $P\left(\mathfrak{h}_{n}^{*}\right)$, the $\mathbb{C}$-valued polynimial functions on $R$-vector space $\mathfrak{h}_{n}^{*}$ via

$$
(x \cdot p)=p\left(k^{-1} \cdot \alpha\right)
$$

Now, if we identify $P\left(\mathfrak{h}_{n}^{*}\right)$ with the complexified symmetric algebra $S\left(\mathfrak{h}_{n}\right)_{I C}$ then the symmetric product $X_{1} X_{2} \cdots X_{n}$ of $X_{1}, X_{2}, \cdots, X_{n} \in \mathfrak{h}_{n}$ becomes the polynomial $\mathfrak{h}_{n}^{*} \rightarrow \mathbb{C}$ given by

$$
P_{X_{1} \cdots X_{n}}(\alpha)=\alpha\left(k \cdot X_{1}\right) \cdots \alpha\left(k \cdot X_{n}\right)=P_{\left(k \cdot X_{1}\right) \cdots\left(k \cdot X_{n}\right)}(\alpha) \text {. }
$$

Now, define a symmetrization map by

$$
\lambda: S\left(\mathfrak{h}_{n}\right):=P\left(\mathfrak{h}_{n}^{*}\right) \rightarrow U\left(\mathfrak{h}_{n}\right),
$$

with

$$
\begin{aligned}
& (\lambda(p) f)(z, t) \\
& =\left.p\left(\frac{\partial}{\partial u}, \frac{\partial}{\partial v}\right) f\left((z, t) \exp \left(\sum_{j=1}^{n} u_{j} X_{j}+\sum_{j=1}^{n} v_{j} Y_{j}\right)\right)\right|_{u=v=0} \\
& =\left.p\left(\frac{\partial}{\partial u}, \frac{\partial}{\partial v}\right) f\left(z+(u+i v), t+\frac{1}{2} w(z, u+i v)\right)\right|_{u=v=0}
\end{aligned}
$$

Now since $U(n)$ acts on $S\left(\mathfrak{h}_{n}\right)$ and $\mathcal{U}\left(\mathfrak{h}_{n}\right)$ by automorphism and $\tilde{\lambda}$ defined by

$$
\begin{aligned}
& \tilde{\lambda}(p) f(z, t) \\
& =\left.p\left(2 \frac{\partial}{\partial \xi}, 2 \frac{\partial}{\partial \xi}\right) f\left(z+\xi, t+\frac{1}{2} w(z, t)\right)\right|_{\xi=0}
\end{aligned}
$$

induces an algebra map on the associated graded algebras and by induction $[10$, p. 282] the eigenfunctions of $\mathcal{L}$ and $\frac{\partial}{\partial t}$ are eigenfunctions of any element in $\mathcal{U}\left(\mathfrak{h}_{n}\right)^{K}$ we have that the following diagram is commutative.

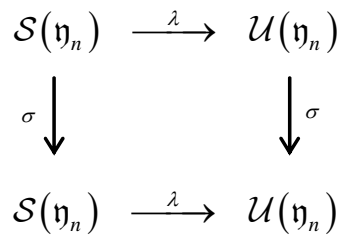

for $\sigma \in \operatorname{Aut}\left(H_{n}\right)$. Since $\lambda$ is a linear isomorphism, it maps $\mathcal{S}\left(\mathfrak{h}_{n}\right)^{K}$ onto $\mathcal{U}\left(\mathfrak{h}_{n}\right)^{K}$. Since the action of $U(n)$ preserves degree on $\mathcal{S}\left(\mathfrak{h}_{n}\right)$, and by [11], if $\left\{1, u_{1}, \cdots, u_{m}\right\}$ generates $\mathcal{S}\left(\mathfrak{h}_{n}\right)^{K}$, then, $\left\{1, \lambda\left(u_{1}\right), \cdots, \lambda\left(u_{m}\right)\right\}$ generates $\mathcal{U}\left(\mathfrak{h}_{n}\right)^{K}$ If $u \in \mathcal{S}\left(\mathfrak{h}_{n}\right)^{K}$, then

$$
u=\sum_{j=1}^{n} P_{j}\left(X_{1}, \cdots, X_{n}, Y_{1}, \cdots, Y_{n}, T_{j}\right)
$$

where the sum is finite and each $P_{j}$ is a polynomial which is $U(n)$-invariant. Thus, the result follows by the fact that the eigenfunctions of $\mathcal{L}$ and $\frac{\partial}{\partial t}$ are the eigenfunctions of $\mathcal{U}\left(\mathfrak{h}_{n}\right)^{K} \quad[12] . \square$

\section{REFERENCES}

[1] G. B. Folland and E. M. Stein, "Estimate for the Complex and Analysis on the Heisenberg Group," Communications on Pure and Applied Mathematics, Vol. 27, No. 4, 1974, pp. 429-522. doi:10.1002/cpa.3160270403

[2] R. Howe, "On the Role of the Heisenberg Group in Harmonic Analysis," Bulletin of the American Mathematical Society, Vol. 3, No. 2, 1980, pp. 821-843. doi:10.1090/S0273-0979-1980-14825-9

[3] E. M. Stein, "Harmonic Analysis: Real Variable Methods, Orthogonality and Oscillatory Integrals," Princeton University Press, Princeton, 1993.

[4] G. B. Folland, "A Fundamental Solution for a Subelliptic Operator," Bulletin of the American Mathematical Society, Vol. 79, No. 2, 1973, pp. 373-376. doi:10.1090/S0002-9904-1973-13171-4

[5] L. P. Rothschild, "Local Solvability of Left-Invariant Differential Operators on the Heisenberg Group," Proceedings of the American Mathematical Society, Vol. 74, No. 2, 1979, pp. 383-388. doi:10.1090/S0002-9939-1979-0524323-X 
[6] M. E. Egwe, "Aspects of Harmonic Analysis on the Heisenberg Group,” Ph.D. Thesis, University of Ibadan, Ibadan, 2010.

[7] H. Lewy, "An Example of a Smooth Linear Partial Differential Operator without Solution," Annals of Mathematics, Vol. 66, No. 2, 1957, pp. 155-158. doi: $10.2307 / 1970121$

[8] U. N. Bassey and M. E. Egwe, "Non-Solvability of Heisenberg Laplacian by Factorization," Journal of Mathematical Sciences, Vol. 21, No. 1, 2010, pp. 11-15.

[9] P. J. Olver, "Application of Lie Groups to Differential
Equations," Graduate Texts in Mathematics, SpringerVerlag, Berlin, 1986.

[10] S. Helgason, "Groups and Geometric Analysis: Integral Geometry, Differential Operators and Spherical Functions," Academic Press Inc., New York, 1984.

[11] V. S. Varadarajan, "Lie Groups, Lie Algebras and Their Representations," Springer-Verlag, Berlin, 1984.

[12] R. Strichartz, "Harmonic Analysis and Radon Transforms on the Heisenberg Group," Journal of Functional Analysis, Vol. 96, No. 2, 1991, pp. 350-406. doi:10.1016/0022-1236(91)90066-E 